Trauma Berufskrankh 2007 · 9[Suppl 3]: S339-S345

DOI 10.1007/s10039-007-1324-2

Online publiziert: 10. November 2007

(c) Springer Medizin Verlag 2007
K.-H. Andro

Landesverband Rheinland-Westfalen

der gewerblichen Berufsgenossenschaften, Düsseldorf

\section{H-Arzt-System und D-Arzt-Aufgaben} Sind die Unterschiede noch sinnvoll?
Die Frage, ob die Unterschiede zwischen D- und H-Arzt-Verfahren noch sinnvoll sind, lässt den Verdacht aufkommen, dass es möglicherweise gar keinen Unterschied zwischen den beiden Verfahren mehr gibt. Bevor die Frage nach der „Sinnhaftigkeit“ $\mathrm{zu}$ beantworten ist, sind zunächst die generellen Unterschiede, aber auch die Gemeinsamkeiten der beiden Heilverfahren darzustellen.

\section{Geschichtliche Entwicklung des D- und H-Arzt-Verfahrens}

Mit den Richtlinien für die berufsgenossenschaftliche Heilfürsorge aus dem Jahr 1925 schufen die berufsgenossenschaftlichen Spitzenverbände die Grundlage für eine sachgemäße Heilfürsorge für das berufsgenossenschaftliche Heilverfahren. Dabei wurde u. a. das Durchgangsarztverfahren (D-Arzt-Verfahren) installiert.

Der D-Arzt beurteilte seinerzeit bereits, ob die Fürsorge der Krankenkasse ausreicht oder ob besondere Heilmaßnahmen notwendig sind - Kriterien, die man in ähnlicher Weise noch immer im Vertrag Ärzte/Unfallversicherungsträger wiederfindet. Schon damals musste der Durchgangsarzt in der Beurteilung und Behandlung von Unfallverletzten besonders erfahren, fachärztlich ausgebildet (Chirurg oder Orthopäde) und ausschließlich fachärztlich tätig sein. Durch die für die damalige Zeit sehr hohen Anforderungen der unfallmedizinischen Ausbildung, Facharzttätigkeit, Praxiseinrichtung und Dienstbereitschaft, besonders bei den niedergelassenen Ärzten, und der damit ver- bundenen noch geringen Anzahl an DÄrzten, wurde - bedingt durch die Fassung des Unfallneuregelungsgesetzes - ab 01.01.1964 die Grundlage für das H-ArztVerfahren gelegt, das alle fachlich befähigten und entsprechend ausgestatteten Ärzte an der Durchführung der berufsgenossenschaftlichen Heilbehandlung, also dem neuen H-Arzt-Verfahren, beteiligen sollte [1].

Die ersten $\mathrm{H}$-Arzt-Richtlinien sahen keine besondere Facharzteigenschaft vor. Der - im Regelfall - niedergelassene Hausarzt musste im Rahmen seiner Ausund Fortbildung über unfallmedizinische Kenntnisse verfügen. Seine Praxis musste die notwendigen Ausstattungsmerkmale, wie eine eigene Röntgenanlage und getrennte Räumlichkeiten für septische und aseptische Eingriffe, aufweisen. So wurde das Durchgangsarztverfahren durch die Hinzunahme des H-Arzt-Verfahrens ergänzt, um ein flächendeckendes medizinisches Versorgungssystem für die Unfallverletzten der gesetzlichen Unfallversicherung $\mathrm{zu}$ installieren.

\section{Aktuelle Situation des D- und H-Arzt-Verfahrens}

Die Landesverbände der gewerblichen Berufsgenossenschaften haben bundesweit über 3500 niedergelassene sowie an Kliniken tätige Ärzte vertraglich in das Durchgangsarztverfahren eingebunden. Daneben sind etwa 3200 Ärzte bundesweit am H-Arzt-Verfahren beteiligt.

Der Durchgangsarzt muss zum Führen der deutschen Facharztbezeichnung
"Chirurgie“ oder „Orthopädie und Unfallchirurgie“ berechtigt und als solcher niedergelassen oder an einem Krankenhaus oder an einer Klinik fachlich und fachlich-organisatorisch weisungsfrei tätig sein. Er muss darüber hinaus - und dies ist der gravierende Unterschied zum H-Arzt-Verfahren - über die deutsche Schwerpunktbezeichnung „Unfallchirurgie“ oder die Zusatzbezeichnung „Spezielle Unfallchirurgie“ verfügen.

Für die Beteiligung eines Bewerbers am H-Arzt-Verfahren ist der Facharzt für Orthopädie bzw. Facharzt für Orthopädie und Unfallchirurgie ausreichend. Ersatzweise kann er nach seiner Approbation eine mindestens 2-jährige unfallmedizinische Tätigkeit in einer mit einem Durchgangsarzt besetzten Krankenhausabteilung nachweisen. Im Regelfall werden derzeit von den Landesverbänden der gewerblichen Berufsgenossenschaften überwiegend niedergelassene Orthopäden am H-Arzt-Verfahren beteiligt.

Ein wesentlicher Unterschied zwischen den beiden Verfahren ist die Verpflichtung des Durchgangsarztes zur unfallärztlichen Bereitschaft, die mindestens in der Zeit von Montag bis Freitag von 08.0018.oo Uhr und Samstag mit der Möglichkeit durchgangsärztlicher Vertretungsregelung von 08.00-13.00 Uhr zu gewährleisten ist. Eine vergleichbare Verpflichtung gibt es für den H-Arzt nicht.

Die H-Arzt-Beteiligung wird seit dem 01.01.2006 nur noch an niedergelassene Ärzte vergeben. Zwar besteht sowohl für die d-als auch für die h-ärztliche Tätigkeit die Verpflichtung der persönlichen Aus- 


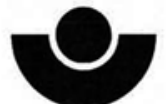

Hauptverband

der gewerblichen

Berufsgenossenschaften e.V.

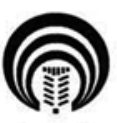

Bundesverband

der landwirtschaftlichen

Berufsgenossenschaften e. v.

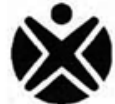

Bundesverband

der Unfallkassen e. V. (BUK)

\section{Anforderungen der gesetzlichen Unfallversicherungsträger nach § 34 SGB VII zur Beteiligung am Durchgangsarztverfahren \\ (in der Fassung vom 1. Januar 2005) \\ aufgestellt von dem Hauptverband der gewerblichen Berufsgenossenschaften, dem Bundesverband der landwirtschaftlichen} Berufsgenossenschaften und dem Bundesverband der Unfallkassen

\section{Präambel}

Am Durchgangsarztverfahren wird ein Arzt beteiligt, der

1.1 gewăhrleistet, dass Qualităt und Wirksamkeit der Leistungen zur Heilbehandlung und Rehabilitation dem allgemein anerkannten Stand der medizinischen Erkenntnisse entsprechen und den medizinischen Fortschritt berücksichtigen,

1.2 über die unter 2. - 4. genannte fachliche Befăhigung personelle und săchliche Ausstattung verfügt,

1.3 persőnlich geeignet ist und

1.4 zur Öbernahme der Pflichten nach 5. bereit ist.

\section{Fachliche Befähigung}

2.1 Der Durchgangsarzt muss zum Führen der deutschen Facharztbezeichnung "Chirurgie" oder "Orthopädie und Unfallchirurgie" berechtigt und als solcher niedergelassen oder an einem Krankenhaus oder einer Klinik fachlich und fachlich-organisatorisch weisungsfrei tătig sein. Er muss darüber hinaus äber die deutsche Schwerpunktbezeichnung „Unfallchirurgie" oder über die Zusatzbezeichnung „Spezielle Unfallchirurgie“ verfügen.

2.2 Ein Arzt, der die Vorraussetzungen nach 2.1 erfült, diese aber im Ausland erworben hat, muss für die Beteiligung als Durchgangsarzt zum Enwerb der Kenntnisse nach 2.3.1 und 2.3.2 nach der Facharztanerkennung ein Jahr bei einem Durchgangsarzt tătig gewesen sein.

\subsection{Erforderlich sind ferner}

2.3.1 eingehende Erfahrungen im durchgangsărztlichen Berichtswesen und in der Gutachtenerstellung,

2.3.2 eingehende Erfahrungen in der Einleitung von $\mathrm{Maß}$ nahmen zur Teilhabe am Arbeitsleben und am Leben in der Gemeinschaft.

2.3.3 Teilnahme an einem Seminar zur Einführung in die Durchgangsarzttätigkeit, das nicht länger als zwei Jahre zuruck liegt

2.3.4 eine nach der Facharzt-Weiterbildung ausgeübte unfallchirurgische Tătigkeit, die nicht länger als drei Jahre unterbrochen worden ist, es sei denn, der Bewerber weist noch genügende unfallchirurgische Kenntnisse nach.

\section{Personelle Ausstattung}

Es mũssen mindestens zwei medizinische Assistenzkräfte stăndig anwesend sein, davon mindestens eine mit abgeschlossener Ausbildung. Werden physiotherapeutische Leistungen in der Praxis des Arztes erbracht, muss zusătzlich eine entsprechende Fachkraft vorhanden sein.

\section{Sächliche Ausstattung}

4.1 Die hygienischen Anforderungen an die baulichfunktionelle und betrieblich-organisatorische Gestaltung richten sich entsprechend der besonderen Aufgabenstellung in der unfallchirurgischen Versorgung nach den Bestimmungen des Infektionsschutzgesetzes (IISG) und den auf seiner Grundlage entwickelten „Anforderungen der Hygiene bei Operationen und anderen invasiven Eingriffen " des Robert-Koch-Instituts, Berlin (RKI-Empfehlungen) (S. $644 \mathrm{ff}$ Bundesgesundheitsblatt 8/2000). Zu beachten ist Punkt 5 (Ambulante Operationen) der RKI-Empfehlungen.

Für die Mindestanforderungen an die bauliche, apparativtechnische und hygienische Ausstattung gilt ergänzend die „Richtlinie der Bundesärztekammer zur Qualitătssicherung ambulanter Operationen" in der jeweils gültigen Fassung.

4.2 Die Praxis soll für nicht gehfähige Unfallverletzte zugänglich und entsprechend ausgestattet sein.

4.3 Neben ausreichenden Untersuchungs- und Behandlungsräumen müssen mindestens vorhanden sein:

4.3.1 Zwei Eingriffsräume für invasive Eingriffe, getrennt für Eingriffe bestimmten Kontaminationsgrades

4.3.2 Umkleidemöglichkeit für das Personal mit Waschbecken und Möglichkeit zur Hăndedesinfektion und zur Entsorgung (in Zuordnung zu den Eingriffsrăumen)

4.3.3 Gerăte-, Vorrats- und Sterilisationsraum (mit normenentsprechender Sterilisationsmöglichkeit), Aufbereitungsbereich

4.3.4 Umkleidebereich für Patienten

4.3.5 Ruheraum für Patienten

4.3.6 Röntgenraum mit einer Rőntgenanlage mindestens der Anwendungsklasse II der Röntgen-Apparate-Richtlinien der Kassenärztlichen Vereinigung

\subsubsection{Wartezone}

4.3.8 ausreichende Einrichtungen zur Archivierung

5 Pflichten

5.1 Der Durchgangsarzt verpflichtet sich, die durchgangsärztliche Tătigkeit in Öbereinstimmung mit den Regelungen und unter Anwendung des Vertrages Ârzte/Unfallversicherungsträger (Årztevertrag) in der jeweils geltenden Fassung auszuüben.

Der Durchgangsarzt verpflichtet sich ferner:

5.2 die durchgangsărztliche Tătigkeit persönlich und unter Beachtung der Grundsătze der Sparsamkeit und Wirtschaftlichkeit auszuüben,

Abb. $1 \Delta$ Anforderungen der gesetzlichen Unfallversicherungsträger nach §34 SGB VII zur Beteiligung am Durchgangsarztverfahren, in der Fassung vom 01.01.2005 [2] 
5.3 unfallarztliche Bereitschaft mindestens in der Ze Montag bis Freitag von 8.00 bis 18.00 Uhr und Samstag mit der Moglichkert durchgangsarztlicher Vertretungsregelungen - von 8.00 bis $13.00 \mathrm{Uhr} z \mathrm{zu}$ gewahrleisten,

5.4 die fur die Unfallversicherungsträger erforderlichen Dokumentationsarbeiten, Begutachtungen sowie Berichterstattungen fristgerecht durchzufüren und insbesondere Durchgangsarztberichte unverzöglich zu erstatten,

5.5 zur Teilnahme am elektronischen Datenaustausch zwischen Leistungserbringern und Unfallversicherungsträgern,

5.6 ârztliche Unterlagen einschl. Krankenblatter, Rontgenaufnahmen mindestens 15 Jahre aufzubewahren,

5.7 an Maßnahmen der gesetzlichen Unfallversicherungstrager zur Qualitatssicherung und deren Umsetzung mitzuwirken

5.8 Arbeitsunfallverletzte mit einer Verletzung nach dem Verletzungsartenverzeichnis unverzugglich einem am Verletzungsartenverfahren beteiligten Krankenhaus oder Arzt zu aberweisen,

5.9 die für die Versorgung Arbeitsunfallverletzter erforderliche Ausstattung der Praxis/des Krankenhauses stets auf dem aktuellen Stand der medizinischen und medizinisch-technischen Entwicklung zu halten,

5.10 zur stăndigen unfallchirurgischen Fortbildung und zur Teilnahme an mindestens einer unfallchirurgischen Fortbildungsveranstaltung pro Jahr,

5.11 zur Abgabe einer Statistik uber die Durchgangsarzttatigkeit fur die Unfallversicherungstrăger bis zum 15 . Feb-
ruar eines jeden Jahres an den zustandigen Landesverband der gewerblichen Berufsgenossenschaften,

5.12 jede Anderung in den die Tatigkeit betreffenden Verhältnissen umgehend dem zustăndigen Landesverband der gewerblichen Berufsgenossenschaften mitzuteilen (z. B. Praxisverlegung, răumliche Praxisumgestaltung, Ändenung der Rechtsform, Umstrukturierung der Klinik).

5.13 jederzeit durch den Landesverband der gewerblichen Berutsgenossenschaften die Erfullung der Anforderungen aberprufen zu lassen

5.14 Aufforderungen der Unfallversicherungstrăger im Zusammenhang mit der Steuerung des Heilverfahrens nachzukommen,

5.15 die Reha-Berater/Berufshelfer Unfallversicherungsträger zu unterstotzen.

\section{Beteiligung}

6.1 Die Beteiligung am Durchgangsarztverfahren erfolgt auf Antrag des Arztes durch offentlich-rechtlichen Vertrag gem. § 53 SGB X mit dem zustăndigen Landesverband der gewerblichen Berufsgenossenschaften.

An einem Standort eines Krankenhauses kőnnen nicht mehrere Durchgangsärzte beteiligt werden.

Die Beteiligung endet

6.2 mit Vollendung des 68. Lebensjahres,

6.3 wenn in einem Zeitraum von funf Jahren im Jahresdurchschnitt nicht mehr als 150 Arbeitsunfallverletzte von dem Durchgangsarzt erstversorgt wurden,

6.4 bei Praxisverlegung oder Praxisaufgabe,

6.5 bei Ausscheiden des Durchgangsarztes aus den Diensten des Krankenhauses, in dem die D-Arzt-Tatigkeit ausgeübt wird,

6.6 bei Kündigung wegen wiederholter Pflichtverletzung.

6.7 bei Kundigung nach Maßgabe des § 59 SGB X.

Abb. $1 \Delta$ Fortsetzung 


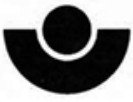

Hauptverband der gewerblichen Berufsgenossenschaften e.V.

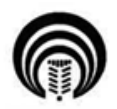

Bundesverband der landwirtschaftlichen Berufsgenossenschaften $e . V$

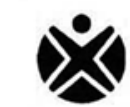

Bundesverband der Unfallkassen e. V. (BUK)

Anforderungen der gesetzlichen Unfallversicherungsträger nach § 34 SGB VII zur Beteiligung am H-Arzt-Verfahren

(in der Fassung vom 1.1.2006)

aufgestellt vom Hauptverband der gewerblichen Berufsgenossenschaften, dem Bundesverband der landwirtschaftlichen Berufsgenossenschaften und dem Bundesverband der Unfallkassen

\section{Präambel}

Am H-Arzt-Verfahren werden nur

niedergelassene

Árzte in ihrer Praxis beteiligt, die

1.1 gewahrleisten, dass Qualităt und Wirksamkeit der Leistungen zur Heilbehandlung und Rehabilitation dem allgemein anerkannten Stand der medizinischen Erkenntnisse entsprechen und den medizinischen Fortschritt berücksichtigen,

1.2 aber die unter 2-4 genannte fachliche Befahigung. personelle und săchliche Ausstattung verfogen,

1.3 persönlich geeignet sind

1.4. zur Öbernahme der Pflichten nach 5 bereit sind.

2 Fachliche Befăhigung

2.1 Der Arzt, der nicht Facharzt fur Orthopadie und Unfallchirurgie ist, muss nachweisen, dass in seiner Zeit der ărztlichen Tatigkeit nach der Approbation eine mindestens zweijahrige unfallmedizinische Tatigkeit in einer mit einem Durchgangsarzt besetzten Krankenhausabteilung enthalten ist.

2.2 Von einer Tatigkeit bei einem niedergelassenen Durchgangsarzt oder von einer Tatigkeit in einer orthopadiDurchgangsarzt oder von einer Tatigkeit in einer orthopadischen Klinik oder orthopâdischen Abteilung eines Krankensche Tatigkeit nach 2.1 angerechnet werden, soweit sie sche Tatigkeit nach 2.1 angerechnet wer.
nach der Approbation absolviert worden ist.

2.3 Bei einem Facharzt für Orthopådie kann auf die zwe Jahre nach 2.1 das nach den Weiterbildungsordnungen de Landesărztekammern abzuleistende eine Jahr Chirurgie angerechnet werden, wenn es ohne fakultative Anrechnungszeiten und nach der Approbation absolviert worden ist.

2.4 Sollen Anrechnungszeiten nach 2.2 und 2.3 berucksichtigt werden, die im Ausland absolviert wurden, muss der Arzt zum Erwerb der Kenntnisse nach 2.5.1 und 2.5.2 eine mindestens 6-monatige Tatigkeit in einer mit einem Durchgangsarzt besetzten Krankenhausabteilung nachweisen.

2.5 Erforderlich sind ferner

2.5.1 eingehende Erfahrungen in der fur die Unfallversicherungsträger erforderlichen Dokumentation und Berichtcherungstrăger erforderlichen Dokumenta
erstattung und in der Gutachtenerstellung.

2.5.2 Erfahrungen in der Einleitung von Maßnahmen zur Teilhabe am Arbeitsleben und am Leben in der Gemeinschaft,

2.5.3 Kenntnisse in der erforderlichen Röntgentechnik und Röntgendiagnostik,

2.5.4 Teilnahme an einem Seminar zur Einfuhrung in die H-Arzt-Tatigkeit,
2.5 .5 eine nach der Approbation ausgeúbte unfallmedizinische oder operativ-orthopaddische Tatigkeit, die nicht lannger als drei Jahre unterbrochen worden ist, es sei denn, der Bewerber weist noch genagende unfallmedizinische Kenntnisse nach.

3 Personelle Ausstattung

Es müssen zwei medizinische Assistenzkrafte in der Praxis sein.

4 Săchliche Ausstattung

- Warteraum

Behandlungsraum

- zwei Eingriffsraume fur invasive Eingriffe getrennt fur Eingriffe bestimmten Kontaminationsgrades

Eingriffe bestimmten Kontaminationsgrades
Róntgenanlage mindestens der Anwendungsklasse II Röntgenanlage mindestens der Anwendungsklasse II
der Rontgenapparate-Richtlinien der Kassenărztlichen der Rontgenapparate-

- normentsprechende Sterilisationsmoglichkeit

ausreichende Ausstattung mit Instrumenten for die Behandlung von Unfallverletzten

- ausreichende Einrichtung zur Archivierung

5 Pflichten

5.1 Der $\mathrm{H}$-Arzt verpflichtet sich, die $\mathrm{H}$-ârztliche Tatigkeit in Öbereinstimmung mit den Regelungen und unter Anwendung des Vertrages Árzte/Unfallversicherungsträger in der jeweils geltenden Fassung auszuuben.

Der H-Arzt verpflichtet sich ferner:

5.2 die $\mathrm{H}$-ărztliche Tatigkeit personlich und unter Beachtung der Grundsatze der Sparsamkeit und Wirtschaftlichkeit auszuauben,

5.3 die fur die Unfallversicherungstrager erforderlichen Dokumentationsarbeiten, Begutachtungen sowie Berichterstattungen fristgerecht durchzuführen und insbesondere $\mathrm{H}$-Arzt-Berichte unverzoglich zu erstatten,

5.4 zur Teilnahme am elektronischen Datenaustausch zwischen Leistungserbringern und Unfallversicherungstrăgern,

5.5 ârztliche Unterlagen einschl. Krankenblatter, Rontgenaufnahmen mindestens 15 Jahre aufzubewahren,

5.6 Arbeitsunfallverletzte mit einer Verletzung nach dem Verletzungsartenverzeichnis unverzuglich einem am Verletzungsartenverfahren beteiligten Krankenhaus oder Arzt zu zungsartenverweisen

5.7 die fur die Versorgung Arbeitsunfallverletzter erforderliche Ausstattung der Praxis stets auf dem aktuellen Stand der medizinischer
lung zu halten,

5.8 zur stăndigen unfallmedizinischen Fortbildung und zur

Abb. $2 \Delta$ Anforderungen der gesetzlichen Unfallversicherungsträger nach §34 SGB VII zur Beteiligung am H-Arzt-Verfahren, in der Fassung vom 01.01.2006 [3] 
Teilnahme an mindestens einer unfallmedizinischen Fortbildungsveranstaltung pro Jahr,

5.9 Aufforderungen der Unfallversicherungstrăger im Zusammenhang mit der Steuerung des Heilverfahrens nachzukommen,

5.10 die Reha-Berater/Berufshelfer der Unfallversicherungsträger zu unterstützen,

5.11 zur Abgabe einer Statistik uber die Tatigkeit fur die Unfallversicherungstrager bis zum 15. Februar eines jeden Jahres an den zuständigen Landesverband der gewerblichen Berufsgenossenschaften,

5.12 jede Ånderung in den die H-Arzt-Tătigkeit betreffenden Verhalttnissen umgehend dem zustândigen Landesverband der gewerblichen Berufsgenossenschaften mitzuteilen (z. B. Praxisverlegung, răumliche Praxisumgestaltung, Ánderung der Rechtsform),

5.13 jederzeit durch den Landesverband der gewerblichen Berufsgenossenschaften die Erfullung der Anforderungen überprüfen zu lassen.

\section{Beteiligung}

6.1 Die Beteiligung am H-Arzt-Verfahren erfolgt auf Antrag des Arztes durch offentlich-rechtlichen Vertrag gemäß § 53 SGB X mit dem zuständigen Landesverband der gewerblichen Berufsgenossenschaften.

Die Beteiligung als $\mathrm{H}$-Arzt gemeinsam mit einem Durchgangsarzt in Praxisgemeinschaft bzw. Gemeinschaftspraxis ist nicht zulässig.

\section{Die Beteiligung endet}

6.2 mit Vollendung des 68 . Lebensjahres,

6.3 bei Praxisverlegung oder Praxisaufgabe,

6.4 bei Kündigung wegen wiederholter Pflichtverletzung,

6.5 bei Kündigung nach Maßgabe des § 59 SGB X.

6.6 Öber den Beteiligungsantrag nach 6.1 und über die Kündigung nach 6.4/6.5 entscheidet ein Ausschuss, der sich aus Vertretern der zustăndigen Kassenärztlichen Vereinigung und des zuständigen Landesverbandes der gewerblichen Berufsgenossenschaften zusammensetzt

Abb. $2 \Delta$ Fortsetzung 
Trauma Berufskrankh 2007 · 9[Suppl 3]: S339-S345 DOI 10.1007/s10039-007-1324-2

(c) Springer Medizin Verlag 2007

\section{K.-H. Andro \\ H-Arzt-System und D-Arzt-Aufgaben. Sind die Unterschiede noch sinnvoll?}

\section{Zusammenfassung}

Durch das Zuweisungsverfahren der Kassen- und Hausärzte und die Möglichkeit der Nachschau übernimmt der Durchgangsarzt (D-Arzt) wichtige Aufgaben für die Steuerung und Überwachung des berufsgenossenschaftlichen Heilverfahrens. Der H-Arzt behandelt ausschließlich die Unfallverletzten, die direkt seine Praxis aufsuchen. Ein Zuweisungsverfahren anderer Ärzte an den H-Arzt ist nach dem Vertrag Ärzte/Unfallversicherungsträger nicht vorgesehen. Der Anteil der Unfallverletzten, die durch einen D-Arzt behandelt werden, beträgt etwa $86 \%$, der verbleibende Anteil der Unfallverletzten, die eine unfallchirurgische Versorgung benötigen, wird von $\mathrm{H}$-Ärzten und Allgemeinärzten versorgt. D-Ärzte haben eine unfallärztliche Be- reitschaft in der Zeit von Montag bis Freitag von 08.00-18.00 Uhr und am Samstag mit der Möglichkeit durchgangsärztlicher Vertretungsregelung von 08.00-13.00 Uhr zu gewährleisten. Für die künftige Beteiligung als D-Arzt reicht der Nachweis der Facharztbezeichnung "Orthopädie und Unfallchirurgie" nicht aus, sondern es ist auch die Zusatzbezeichnung „Spezielle Unfallchirurgie” erforderlich.

\section{Schlüsselwörter}

D-Arzt-Verfahren · H-Arzt-Verfahren . Steuerung des berufsgenossenschaftlichen Heilverfahrens - Überwachung des berufsgenossenschaftlichen Heilverfahrens. Qualifikation

\section{Tasks of the two types of doctors available for emergency trauma treatment. Is there any point in continuing to make a difference?}

\begin{abstract}
The procedure in place for health insuranceapproved doctors and general practitioners and the option of checking assign important tasks in the control and monitoring of treatments paid for by the employers' liability insurance associations to the doctor in private practice who is qualified for emergency treatment (D[Durchgangs]-Arzt). The trauma doctor in private practice who has not undertaken the training for this qualification ( $\mathrm{H}-\mathrm{Ar}-$ zt) is only allowed to treat such injured persons as come to his or her practice direct. The contract between doctors and accident insurance payers has no procedure in place for other doctors to refer patients to the $\mathrm{H}$-Arzt. Approximately $86 \%$ of injured patients are treated by a D-Arzt, while the remaining pa-
\end{abstract}

tients who need trauma surgery are treated by an $\mathrm{H}$-Arzt or general practitioner. A D-Ar$\mathrm{zt}$ is obliged to be available on Mondays to Fridays from 08.00 to 18.00 and to provide an on-call service on Saturdays from 08.00 to 13.00 ; it is permissible for the Saturday hours to be entrusted to an officially approved locum. In future a documented qualification in orthopaedics and trauma surgery will no longer be enough to allow a doctor to work as a D-Arzt; the additional specialist qualification in trauma surgery is also required.

\section{Keywords}

D-Arzt procedures $\cdot \mathrm{H}$-Arzt procedures . Control of approved treatment $\cdot$ Monitoring of approved treatment · Qualification übung der Arzttätigkeit, allerdings können die Landesverbände der gewerblichen Berufsgenossenschaften ständige Durchgangsarztvertreter anerkennen, wenn diese über die gleiche fachliche Befähigung wie ein Durchgangarzt verfügen. Diese Vertretungsregelung ist dem H-Arzt nach dem Vertrag Ärzte/Unfallversicherungsträger nicht gegeben.

Sofern der H-Arzt nicht in der Praxis anwesend ist, sind die Unfallverletzten vom Vertreter unverzüglich einem Durchgangsarzt vorzustellen, wenn der Verletzte über den Unfalltag hinaus arbeitsunfähig ist oder die Behandlungsbedürftigkeit voraussichtlich mehr als 1 Woche beträgt. Damit ist das H-Arzt-Verfahren an Krankenhäusern nicht praktikabel.

Hinsichtlich der Überprüfung der sächlichen Ausstattung zur Beteiligung an den Heilverfahren, wie das Vorhalten zweier Eingriffsräume für invasive Eingriffe oder der Röntgenanlage, werden bei der Abnahme durch die Landesverbände der gewerblichen Berufsgenossenschaften zwischen D- und H-Arzt-Praxen keine Unterschiede gemacht.

Die weiteren detaillierten Ausstattungsmerkmale der D- und H-Arzt-Praxen sind den Anforderungen zur Beteiligung am $\mathrm{D}$ - und $\mathrm{H}$-Arzt-Verfahren zu entnehmen $[2,3]$ ( Abb. 1, Abb. 2).

\section{Aktuelle Unterschiede zwischen D- und H-Arzt- Tätigkeit laut Vertrag Ärzte/Unfallversicherungsträger}

Außer der Beachtung des Verletzungsartenverzeichnisses in besonders schweren Behandlungsfällen unterliegen sowohl der D- als auch der H-Arzt keiner Behandlungseinschränkung. Diese schweren Behandlungsfälle sind unverzüglich in ein entsprechendes VAV-Krankenhaus weiterzuleiten [4]. Dies gilt ebenfalls für Behandlungen außerhalb des unfallchirurgisch/orthopädischen Behandlungsbereichs wie der Augen, des HNO-Bereichs oder bestimmten Berufskrankheiten, die besonderen Fachärzten zuzuführen sind.

Eine Vorstellungspflicht des $\mathrm{H}$-Arztes an den D-Arzt ist im Ärztevertrag nicht vorgesehen. Selbstverständlich kann sowohl der D- als auch der H-Arzt zur Klärung der Diagnose oder zur Mitbehand- 
lung andere Ärzte hinzuziehen. Dieses gilt insbesondere für Ärzte anderer Fachrichtungen, wenn bei der Art der Verletzung der Verdacht auf eine Mitbeteiligung eines entsprechenden Organs oder Organsystems besteht. Diese Konsultationsmöglichkeit ist nur den D- und H-Ärzten vorbehalten, ebenso auch die Verordnung von Heilmitteln (KG, EAP, BGSW).

Ärzte, die keine D- oder H-Ärzte sind, haben Unfallverletzte unverzüglich einem Durchgangsarzt vorzustellen, wenn die Unfallverletzung zur Arbeitsunfähigkeit führt oder die Behandlungsbedürftigkeit voraussichtlich mehr als 1 Woche beträgt. Dieses Zuweisungsverfahren ist im $\mathrm{H}$ Arzt-Verfahren nicht vorgesehen.

Der sehr hohe Anteil der erstatteten D-Arzt-Berichte resultiert zum einen aus der Überweisungspflicht der Kassen-/ Hausärzte und zum anderen aus der Verpflichtung der Unternehmer/Arbeitgeber nach $\$ 24$ BGV A 1, unfallverletzte Arbeitnehmer einem Durchgangsarzt vorzustellen. So beträgt die Summe der erstatteten Berichte im Durchgangsarztverfahren (2005: 2.780.207) das 7-Fache der erstatteten Berichte im H-Arzt-Verfahren (2005: 395.461) [5].

Ein weiteres besonderes Merkmal des D-Arzt-Verfahrens ist die Möglichkeit der Nachschau. Dabei handelt es sich um Behandlungsfälle, die der Durchgangsarzt aufgrund der vermeintlich geringeren Schwere zunächst in die allgemeine Heilbehandlung dem Hausarzt überweist. Bei diesen nicht in eigener Behandlung verbleibenden Unfallverletzten hat der Durchgangsarzt Nachschautermine festzusetzen. Bei weiter andauernder bestehender Arbeitsunfähigkeit sind die Unfallverletzten dem Durchgangsarzt zur nochmaligen Beurteilung an dem vorgegebenen Nachschautermin wieder vorzustellen. Diese Möglichkeit fehlt im HArzt-Verfahren. Der H-Arzt behandelt ausschließlich die Unfallverletzten, die in seine Praxis kommen und dort in der Behandlung verbleiben.

Der Anteil der besonderen berufsgenossenschaftlichen Heilbehandlung des H-Arztes, also auch der höheren Abrechnungsmöglichkeit gegenüber der allgemeinen Heilbehandlung, liegt bedingt durch $\$_{35}$ des Vertrags Ärzte/Unfallversicherungsträger bei nur etwa 10\% seiner gesamten berufsgenossenschaftlichen Behandlungsfälle.

\section{Fazit}

Der Arzt mit der spezielleren unfallchirurgischen Ausbildung, also der Durchgangsarzt, versorgt den weitaus größeren Anteil der Unfallverletzten. Durch das Zuweisungsverfahren und die Möglichkeit der Nachschau übernimmt er wichtige Aufgaben für die Steuerung und Überwachung des berufsgenossenschaftlichen Heilverfahrens.

Es liegt nicht im Interesse der Unfallversicherungsträger, den hohen Qualitätsstandard des D-Arzt-Verfahrens durch die neue Weiterbildungsordnung oder auch durch die Zusammenlegung der Facharztbezeichnungen in Frage zu stellen. Daher reicht es nach den zurzeit gültigen D-Arzt-Anforderungen eben nicht aus, die Facharztbezeichnung „Orthopädie und Unfallchirurgie" nachzuweisen, sondern es muss auch die Zusatzbezeichnung „Spezielle Unfallchirurgie“ vorhanden sein.

\section{Korrespondenzadresse}

\section{K.-H. Andro}

Landesverband Rheinland-Westfalen der gewerblichen Berufsgenossenschaften, Kreuzstraße 45, 40210 Düsseldorf

service@duesseldorf.lvbg.de

Interessenkonflikt. Keine Angaben

\section{Literatur}

1. Haep F (1964) Die BG 3

2. HVBG, BLB, BUK (2005) Anforderungen der gesetzlichen Unfallversicherungsträger nach §34 SGB VII zur Beteiligung am Durchgangsarztverfahren, Fassung vom 01.01.2005. HVBG, St. Augustin

3. HVBG, BLB, BUK (2006) Anforderungen der gesetzlichen Unfallversicherungsträger nach $\$ 34$ SGB VII zur Beteiligung am H-Arzt-Verfahren, Fassung vom 01.01.2006. HVBG, St. Augustin

4. DGUV (2005) Verletzungsartenverzeichnis, Fassung vom 01.01.2005. BLB, Kassel

5. Landesverbände der gewerblichen Berufsgenossenschaften: Statistik. http://www.lvbg.de/lv/ pages/statistik 